\title{
Desempenho agronômico de genótipos de amendoim sob cultivo mínimo no Triângulo Mineiro
}

Submetido - 12 jul. $2020 \quad$ Aprovado - 13 out. $2020 \quad$ Publicado - 14 out. 2020
dol http://dx.doi.org/10.17648/sas.v1i1.20

Antonio Carlos Aparecido Filho (D) MGV Agroindustrial Ltda., Iturama, MG, e-mail: antonio.carlos@mgvagroindustrial.com.

Amanda Severino Soares ${ }^{(D)}$

Discente de Agronomia, Universidade Federal do Triângulo Mineiro/UFTM, Iturama, MG, e-mail: amandass.agro@gamil.com.

Programa de Melhoramento do Amendoim - Embrapa, Santo Antônio de Goiás, GO, e-mail:
jair.heuert@embrapa.br. Mariel Gomes da Silva ${ }^{D}$ Discente de Agronomia, Universidade Federal do Triângulo Mineiro/UFTM, Iturama, MG, e-mail: marielgomes.agro@gmail.com.

Programa de Melhoramento do Amendoim - Embrapa, Santo Antônio de Goiás, GO, e-mail: tais.suassuna@embrapa.br.

\section{RESUMO}

Objetivou avaliar desempenho agronômico de genótipos de amendoim sob cultivo mínimo no Triângulo Mineiro. O ensaio foi instalado em uma área de renovação de canavial, em cultivo mínimo, no ano agrícola 2019/20, na região de Iturama-MG. O delineamento experimental utilizado foi em blocos casualizados, contendo quatro repetições e oito tratamentos: as linhagens 18-2101 OL, 17-1253 OL, 18-2133 OL, 18-2136 OL, 18-1952 OL e as cultivares BRS 421 OL, BRS 423 OL, BRS 425 OL, desenvolvidas pelo Programa de Melhoramento do Amendoim da Embrapa. As parcelas foram constituídas por duas linhas de três metros de comprimento, espaçamentos entre linhas de noventa centímetros e intervalo de três metros entre parcelas. Foram avaliados severidade de mancha preta, massa de 100 grãos e produtividade de vagens. As menores severidades de mancha preta foram observadas nas cultivares BRS 421 OL, BRS 423 OL e BRS 425 OL. A maior massa de grãos foi observada na BRS 421 OL (81,8 g), sendo os demais genótipos entre 74,4 e 77,6 g. Os genótipos mais produtivos foram 18-2101 OL, BRS 423 OL, 17-1253 OL, BRS 421 OL e 18-2133 OL.

Palavras-chave: Arachis hypogaea L.; Programa de Melhoramento; Produtividade.

\section{Agronomic performance of peanut genotypes under minimum cultivation in the Triângulo Mineiro}

\begin{abstract}
It aimed to evaluate the agronomic performance of peanut genotypes under minimum cultivation in the Triângulo Mineiro. The trial was installed in a sugarcane renewal area, in minimal cultivation, in the 2019/20 agricultural year, in the Iturama-MG region. The experimental design used was in randomized blocks, containing four replicates and eight treatments: lines 18-2101 OL, 17-1253 OL, 18-2133 OL, 18-2136 OL, 18-1952 OL and cultivars BRS 421 OL, BRS 423 OL, BRS 425 OL, developed by Embrapa's Peanut Breeding Program. The plots were constituted by two lines of three meters in length, spacing between lines of ninety centimeters and an interval of three meters between plots. Severity of black spot, mass of 100 grains and
\end{abstract}


pod yield were evaluated. The lowest severity of black spot was observed in the cultivars BRS $421 O L, B R S 423 O L$ and BRS $425 O L$. The highest grain mass was observed in BRS $421 O L$ $(81.8 \mathrm{~g})$, with the other genotypes between 74.4 and $77.6 \mathrm{~g}$. The most productive genotypes were 18-2101 OL, BRS 423 OL, 17-1253 OL, BRS 421 OL and 18-2133 OL.

Keywords: Arachis hypogaea L.; Improvement Program; Productivity.

\title{
Rendimiento agronómico de genotipos de maní bajo cultivo mínimo en el Triângulo Mineiro
}

\author{
RESUMEN
}

Su objetivo era evaluar el rendimiento agronómico de los genotipos de maní bajo cultivo mínimo en el Triângulo Mineiro. La prueba se instaló en un área de renovación de caña de azúcar, en un cultivo mínimo, en el año agrícola 2019/20, en la región de Iturama-MG. El diseño experimental utilizado fue en bloques aleatorios, que contenían cuatro réplicas y ocho tratamientos: líneas 18-2101 OL, 17-1253 OL, 18-2133 OL, 18-2136 OL, 18-1952 OL y cultivares BRS 421 OL, BRS 423 OL, BRS 425 OL, desarrollado por Embrapa's Peanut Breeding Program. Las parcelas consistían en dos líneas de tres metros de longitud, espaciadas entre líneas de noventa centímetros y un intervalo de tres metros entre parcelas. Se evaluaron la gravedad de la mancha negra, la masa de 100 granos y el rendimiento de la vaina. La menor severidad de la mancha negra se observó en los cultivares BRS 421 OL, BRS 423 OL y BRS 425 OL. La mayor masa de grano se observó en BRS 421 OL (81.8 g), con los otros genotipos entre 74.4 y $77.6 \mathrm{~g}$. Los genotipos más productivos fueron 18-2101 OL, BRS 423 OL, 17-1253 OL, BRS 421 OL y 18$2133 O L$

Palabras clave: Arachis hypogaea L.; Programa de Mejoramiento; Productividad.

\section{Introdução}

O amendoim (Arachis hypogaea L.) é cultivado em sucessão com cana-de-açúcar em áreas de reformas de canaviais, proporcionando diversos benefícios físicos, químicos e biológicos ao solo e para cultura subsequente. Segundo Péres (2013) esse sistema de cultivo diminui os custos de aplicações de fertilizantes por causa das estirpes nativas das bactérias de fixação biológica de nitrogênio, melhoram a ciclagem de nutrientes, aeração e infiltração de água no solo por meio do sistema radicular profundo. Essa rotação promove benefícios as condições do solo, por meio do aumento da disponibilidade de nutrientes, e influenciando no incremento na produção da cana (AMBROSANO et al., 2011).

Além da rotação de culturas proporcionar a quebra do ciclo patógenos-hospedeiro e possíveis diminuições de aplicações de fungicidas e inseticidas. A palhada proporciona a redução de danos ocasionados por ataque do tripes, dificultando o seu deslocamento e consequentemente 
reduz a incidência de plantas com virose na área (SHOLAR et al., 1995). O plantio do amendoim sob palhada de cana crua reduz o revolvimento do solo, a exposição do banco de sementes de plantas daninhas, além de manter a palhada no solo, aumentando a umidade e diminuindo a incidência de plantas daninhas (SOARES, 2014).

Por ser mais exigente que outras culturas anuais em cálcio e magnésio, o cultivo do amendoim requer aplicação de boas quantidades de calcário e gesso. Assim, ao final do cultivo do amendoim, o solo está solo previamente corrigido e preparado para a produção de cana nos anos seguintes, observando-se acréscimo de produtividade até o terceiro corte e possível substituição integral de alguns nutrientes (VITTI et al., 2011). Outro benefício é a redução na população de nematoides do gênero Pratylenchus brachyurus, na cana-de-açúcar após o cultivo de espécies leguminosas como o amendoim (MENDES et al., 2019; AMBROSANO et al., 2011).

Objetivou avaliar desempenho agronômico de genótipos de amendoim sob cultivo mínimo no Triângulo Mineiro, sendo cinco linhagens e três cultivares, desenvolvidas pelo Programa de Melhoramento do Amendoim da Embrapa.

\section{Material e métodos}

O ensaio foi instalado em uma área de renovação de canavial, no ano agrícola 2019/20, na região de Iturama-MG (50 48' 53" W e 19 $41^{\prime}$ ' $02^{\prime \prime}$ $\mathrm{S}$, com altitude de 485 metros). A semeadura foi realizada no dia 24 de outubro de 2019, em cultivo mínimo.

Foi utilizado o delineamento em blocos casualizados, contendo quatro repetições e oito tratamentos: as linhagens $18-2101 \mathrm{OL}, 17-1253 \mathrm{OL}$, 18-2133 OL, 18-2136 OL, 18-1952 OL e as cultivares BRS 421 OL, BRS 423 OL, BRS $425 \mathrm{OL}$ desenvolvidas pelo Programa de Melhoramento do Amendoim da Embrapa. As parcelas foram compostas por duas linhas de três metros de comprimento, espaçamentos entre linhas de noventa centímetros e intervalo de três metros entre parcelas. 
A pulverização de herbicidas, fungicidas e inseticidas (manejo fitossanitário) e aplicação de gesso agrícola e adubações (correção do solo) foram realizadas conforme as recomendações para a cultura e análise de solo da área. $\mathrm{O}$ estande médio estabelecido foi de 15 plantas. $\mathrm{m}^{-1}$. Aos 130 dias após a semeadura (DAE), foi realizada a inversão mecanizada das plantas e após a secagem foram feitas as avaliações de massa de 100 grãos (g) e produtividade de vagens (kg.ha ${ }^{-1}$, sacas.ha ${ }^{-1}$ e sacas.alqueire ${ }^{-1}$ ).

Foi avaliado severidade de mancha preta (Cercosporidium personatum) anterior a inversão de plantas, usando a escala diagramática da incidência com notas de 1 a 9 ao final do ciclo dos genótipos (SUBRAHMANYAM et al., 1982). Os dados obtidos foram submetidos à análise de variância (Teste $F$ ) e as médias dos tratamentos comparadas pelo teste de Scott-Knott a $5 \%$ de probabilidade, por meio do programa computacional SISVAR 5.6 (FERREIRA, 2019).

\section{Resultados e discussão}

Houve diferença significativa para as variáveis severidade de mancha preta (notas), massa de 100 grãos (g) e produtividade de vagens $\left(\mathrm{kg} \cdot \mathrm{ha}^{-1}\right)$ em função dos diferentes genótipos testados. Observa-se que as cultivares BRS 421 OL $(2,5)$, BRS 423 OL $(3,5)$ e BRS 425 OL $(3,5)$ apresentaram as menores notas de severidade de mancha preta quando comparado aos demais, resultando possível tolerância a mancha preta.

As linhagens 18-2133 OL (4,0), 17-1253 OL $(4,5), 18-2136$ OL $(4,5)$ e 18-1952 OL $(4,5)$ obtiveram tolerância intermediária. Por outro lado, somente a linhagem 18-2101 OL $(6,0)$ apresentou-se com a maior nota de severidade, sendo-a mais suscetível nas condições experimentais. Martins et al. (2019), observaram a linhagem 18-2101 OL (8,4) com a maior severidade quando comparada aos mesmos genótipos em Santo Antônio de Goiás-GO.

Na massa de 100 grãos, a maior massa foi obtida na cultivar BRS 421 OL $(81,8$ g). Observa-se massas intermediárias nos genótipos 17-1253 
OL $(77,6 \mathrm{~g}), 18-1952$ OL $(77,6 \mathrm{~g})$ e $18-2133$ OL $(77,1 \mathrm{~g})$. Por fim, os genótipos 18-2101 OL (75,6 g), BRS 423 OL $(75,4$ g), BRS 425 OL $(74,7 \mathrm{~g})$ e 18-2136 OL $(74,4 \mathrm{~g})$ apresentaram as menores massas. Estes resultados representam diversidade na granulometria dos grãos, quesito este satisfatório para a possibilidade da produção de grãos atender a diferentes exigências de mercado. Domenici et al. (2018) obtiveram massas correlatas com as cultivares BRS $423 \mathrm{OL}(76,3 \mathrm{~g})$ e BRS $425 \mathrm{OL}(74,0 \mathrm{~g})$ em FrutalMG. Martins et al. (2019), observaram resultados semelhantes com as linhagens 18-2136 OL (73,9 g), 18-2101 OL (76,7 g), 17-1253 OL (75,4 g), $18-2133$ OL $(75,6 \mathrm{~g})$ e $18-1952 \mathrm{OL}(78,4 \mathrm{~g})$.

Tabela 1. Severidade de mancha preta (notas), massa de 100 grãos (g) e produtividade de vagens ( $\mathrm{kg} \cdot \mathrm{ha}^{-1}$, sacas.ha ${ }^{-1}$ e sacas.alqueire ${ }^{-1}$ ) em função de diferentes genótipos de amendoim. Iturama-MG, safra 2019/20.

\begin{tabular}{|c|c|c|c|c|c|}
\hline \multirow{2}{*}{ Genótipos } & \multirow{2}{*}{$\begin{array}{c}\text { Severidade de } \\
\text { mancha preta }\end{array}$} & \multirow{2}{*}{$\begin{array}{c}\text { Massa de } \\
100 \text { grãos } \\
\text { (g) }\end{array}$} & \multicolumn{3}{|c|}{ Produtividade de vagens } \\
\hline & & & $\left(\mathrm{kg} \cdot \mathrm{ha}^{-1}\right)$ & $\left(\right.$ sacas.ha $\left.^{-1}\right)$ & $\left(\right.$ sacas.alqueire $\left.^{-1}\right)$ \\
\hline $18-2101 \mathrm{OL}$ & $6,0 \mathrm{c}$ & $75,6 \mathrm{c}$ & $7.937,4 \mathrm{a}$ & 317,5 & 768,3 \\
\hline BRS $423 \mathrm{OL}$ & $3,5 \mathrm{a}$ & $75,4 \mathrm{c}$ & $7.570,0 \mathrm{a}$ & 302,8 & 732,8 \\
\hline $17-1253 \mathrm{OL}$ & $4,5 \mathrm{~b}$ & $77,6 \mathrm{~b}$ & $7.365,8 \mathrm{a}$ & 294,6 & 713,0 \\
\hline BRS $421 \mathrm{OL}$ & $2,5 \mathrm{a}$ & $81,8 \mathrm{a}$ & $6.982,1 \mathrm{a}$ & 279,3 & 675,9 \\
\hline $18-2133 \mathrm{OL}$ & $4,0 \mathrm{~b}$ & $77,1 \mathrm{~b}$ & $6.970,1 \mathrm{a}$ & 278,8 & 674,7 \\
\hline 18-2136 OL & $4,5 \mathrm{~b}$ & $74,4 \mathrm{c}$ & $6.088,5 \mathrm{~b}$ & 243,5 & 589,4 \\
\hline BRS 425 OL & $3,5 \mathrm{a}$ & $74,7 \mathrm{c}$ & $5.833,2 \mathrm{~b}$ & 233,3 & 564,7 \\
\hline 18-1952 OL & $4,5 b$ & $77,6 \mathrm{~b}$ & $5.758,3 \mathrm{~b}$ & 230,3 & 557,4 \\
\hline Média & 4,1 & 76,8 & $6.813,2$ & 272,5 & 659,5 \\
\hline C.V. (\%) & 18,5 & 1,1 & 13,9 & - & - \\
\hline $\operatorname{Pr}>\mathrm{Fc}$ & $>0,0001^{*}$ & $>0,0001^{*}$ & $0,0187^{\star}$ & - & - \\
\hline
\end{tabular}

Os genótipos 18-2101 OL (7.937,4 kg.ha-1), BRS 423 OL (7.570,0 $\left.\mathrm{kg} \cdot \mathrm{ha}^{-1}\right), 17-1253 \mathrm{OL}\left(7.365,8 \mathrm{~kg} \cdot \mathrm{ha}^{-1}\right)$, BRS $421 \mathrm{OL}\left(6.982,1 \mathrm{~kg} \cdot \mathrm{ha}^{-1}\right)$ e $18-$ $2133 \mathrm{OL}\left(6.970,1 \mathrm{~kg}^{-h^{-1}}\right)$ obtiveram as maiores produtividades de vagens, sendo superiores a 270 sacas.ha-1 ou 670 sacas.alqueire ${ }^{-1}$. Verifica-se que os genótipos 18-2136 OL (6.088,5 kg.ha-1), BRS $425 \mathrm{OL}\left(5.833,2 \mathrm{~kg} \cdot \mathrm{ha}^{-1}\right)$ e 18-1952 OL (5.758,3 kg.ha-1) apresentaram as menores. Observa-se que dentre as cultivares, a BRS $423 \mathrm{OL}$ e BRS $421 \mathrm{OL}$ produziram quantia superior estatisticamente ao BRS $425 \mathrm{OL}$. De acordo com Suassuna et al. (2020), que relatam a adaptação dos cultivares BRS 423 OL e BRS 421 OL 
ao bioma Cerrado. De modo generalizado todos os genótipos obtiveram produtividade superior à estimativa média do estado de Minas Gerais de $3.516 \mathrm{~kg}^{-\mathrm{ha}^{-1}}$ (CONAB, 2020).

\section{Conclusões}

As menores severidades de mancha preta foram observadas nas cultivares BRS $421 \mathrm{OL}$, BRS $423 \mathrm{OL}$ e BRS $425 \mathrm{OL}$. A maior massa de grãos foi observada na BRS $421 \mathrm{OL}(81,8 \mathrm{~g})$, sendo os demais genótipos entre 74,4 e 77,6 g. Os genótipos mais produtivos foram o 18-2101 OL, BRS 423 OL, 17-1253 OL, BRS 421 OL e 18-2133 OL.

\section{Agradecimentos}

Os autores agradecem a MGV Agroindustrial pela cooperação técnica e financeira que viabilizou a execução deste estudo. Ao produtor rural Antonio Carlos Aparecido por apoiar na condução do experimento. Este experimento faz parte do Programa de Melhoramento do Amendoim da Embrapa vinculado ao projeto (SEG 20.18.01.021.00). 


\section{Referências}

AMBROSANO, E. J.; CANTARELLA, H.; AMBROSANO, G. M. B.; SHAMMASS, E. A.; DIAS, F. L. F.; ROSSI, F.; TRIVELIN, P. C. O.; MURAOKA, T.; SACHSM, R. C. C.; AZCÓN, R. Produtividade de cana-deaçúcar após cultivos de leguminosas. Bragantia, v. 70, n. 4, p. 810-818, 2011.

CONAB. Acompanhamento da Safra Brasileira de grãos. Safra 2019/20 Quinto levantamento, v. 7, n. 5, p. 1-112, fev. 2020. Disponível em: <https://www.conab.gov.br/info-agro/safras>. Acesso em: 23 jun. 2020.

DOMENICI, M. G.; ZACHARIAS, A. O.; GUIRALDELLI, E. H.; HEUERT, J.; SUASSUNA, T. M. F. Desempenho de genótipos de amendoim no Triângulo Mineiro. In: Anais do Encontro Sobre a Cultura do Amendoim, 15., 2018, Jaboticabal. Anais eletrônicos... Campinas: GALOÁ, 2018. Disponível em: $<$ https://proceedings.science/encontro-amendoim-2018/papers/desempenhode-genotipos-de-amendoim-no-triangulo-mineiro>. Acesso em: 23 jun. 2020.

FERREIRA, D. F. SISVAR: um sistema de análise de computador para efeitos fixos projetos de tipo de partida dividida. Revista Brasileira de Biometria, v. 37, n. 4, p. 529-535, 2019.

MARTINS, K. B. B.; RODRIGUES, L. L.; HEUERT, J.; XAVIER, M. F. N.; SUASSUNA, T. M. F.; BETIOL, R. A. B. Desempenho agronômico de novas linhagens de amendoim no Cerrado. In: Anais do Encontro Sobre a Cultura do Amendoim, 16., 2019, Jaboticabal. Anais eletrônicos... Campinas: GALOÁ, 2019. Disponível em: <https://proceedings.science/encontroamendoim-2019/papers/desempenho-agronomico-de-novas-linhagens-deamendoim-no-cerrado>. Acesso em: 23 jun. 2020.

MENDES, A. C. S.; FREIRE, L. L.; PUERARI, H. H.; HEUERT, J.; ROCHA, M. R. Hospedabilidade de cultivar de amendoim A Pratylenchus brachyurus. In: Anais do Congresso Brasileiro de Nematologia, 36., 2019, Caldas Novas. Resumos... Caldas Novas: CBN, 2019. Disponível em: $<$ http://www.infobibos.com/anais/cbn/36/Resumos/Resumo36CBN_0168.pdf >. Acesso em 23 jun. 2020.

PÉRES, J. S. G. Sucessão de culturas e adubação nitrogenada em cana soca: Efeitos nos atributos químicos do solo, na produtividade e na qualidade da cultura. Dissertação (Mestrado em Agronomia). Universidade Estadual Paulista "Julio de Mesquita Filho", Faculdade de Ciências Agrárias e Veterinárias, 32 p, 2013.

SHOLAR, J.; MOZINGO, R. W.; BEASLEY JUNIOR, J. Peanut cultural practices. In: PATEE, H. E.; STALKER, H. T. (Ed.). Advances in peanut science. Stillwater: American Peanut Research and Education Society, 1995. p.354-382. 
SOARES, M. B. B. Sistemas de cultivo em área de reforma de cana-deaçúcar e a sucessão de culturas na composição da comunidade infestante. Dissertação (Mestrado em Agronomia). Universidade Estadual Paulista "Julio de Mesquita Filho", Faculdade de Ciências Agrárias e Veterinárias, 70 p, 2014.

SUASSUNA, T. M. F. et al. 'BRS 421' and 'BRS 423': high oleic peanut cultivars for production in Brazil. Crop Breeding Applied Biotechnology, v. 20, n. 1, e28932018, 2020.

SUBRAHMANYAM, P.; MCDONALD, D.; GIBBONS, R. W.; NIGAM, S. N.; NEVILL, D. J. Resistance to rust and late leaf spot diseases in some genotypes of Arachis hypogaea. Peanut Science, v. 9, p. 9-14, 1982.

VITTI, A. C.; FRANCO, H. C. J.; TRIVELIN, P. C. O.; FERREIRA, D. A.; OTTO, R.; FORTES, C.; FARONI, C. E. Nitrogênio proveniente da adubação nitrogenada e de resíduos culturais na nutrição da cana-planta. Pesquisa Agropecuária Brasileira, v. 46, n. 3, p. 287-293, 2011. 